\title{
Evapotranspiração e coeficiente de cultura do cafeeiro irrigado por pivô central ${ }^{1}$
}

\author{
Antonio C. da Silva ${ }^{2}$, Luiz A. Lima ${ }^{2}$, Adão W. P. Evangelista ${ }^{3}$ \& Carla P. Martins ${ }^{4}$
}

\begin{abstract}
RESUMO
Objetivou-se, neste trabalho, estimar a evapotranspiração e o coeficiente de cultivo (Kc) do cafeeiro "Rubi" irrigado por pivô central, através do método do balanço hídrico. O experimento foi desenvolvido na área experimental da Universidade Federal de Lavras e o delineamento experimental adotado foi o de blocos casualizados, com 6 tratamentos e 3 repetições. Os tratamentos corresponderam à lâmina de água aplicada em função de percentagens de valores de Kc, ou seja, 60, 80, 100, 120 e 140\% do valor de Kc, além de um tratamento sem irrigação (testemunha), totalizando 18 parcelas; em cada parcela se consideraram 8 plantas úteis. Os valores de evapotranspiração da cultura (ETC) do café encontrados foram de 3,46, 7,14 e 5,41 mm, para os períodos de 01/04 a 31/07 (B), 01/08 a 31/10 (C) e 01/11 a 31/ 12 (D) do ano de 2007, respectivamente. Nos períodos de 01/01 a 31/03 (A) e de 01/04 a 18/05 (B) do ano de 2008, os valores de ETc foram de 5,57 e 3,07 $\mathrm{mm} \mathrm{d}^{-1}$, respectivamente, e um valor médio de 4,93 $\mathrm{mm} \mathrm{d}^{-1}$. Os valores de Kc do café encontrados para os períodos A, B, C e D, foram de 1,32, 1,06, 1,61, 1,25 , respectivamente.
\end{abstract}

Palavras-chave: irrigação, aspersão, café, balanço hídrico

\section{Evapotranspiration and crop coefficient of irrigated coffee with a pivot}

\begin{abstract}
Evapotranspiration and crop coefficient (KC) were estimated through water balance for coffee crop (cultivar Rubi), irrigated by center pivot. The experiment was carried out at the experimental area of Federal University of Lavras. Randomized block was used as statistical design, with 6 treatment and 3 replicates. The treatment levels were set by water depth applied as percentage of Kc values predefined and used by farmers in the region, established as $60,80,100,120$ e $140 \%$ of the predefined Kc value for a given period. The experiment included a control (non-irrigated), totalizing 18 plots, being considered in each one 8 effective plants for measurements. According to results obtained for soil water potential, the best treatment levels indicate coffee evapotranspiration of 3.46, 7.14 and $5.41 \mathrm{~mm}$, for periods, B (April 4 to July 31), C (August 1 to October 31) and D (November 1 to December 31) during 2007, respectively. For periods A (January 1 to March 31 ) and B in 2008, the ETc values were 5.57 and $3.07 \mathrm{~mm} \mathrm{~d}^{-1}$, respectively, with average of $4.93 \mathrm{~mm} \mathrm{~d}^{-1}$. The best Kc values found were 1.32, 1.06, 1.61 and 1.25 for periods $A, B$, C and D, respectively.
\end{abstract}

Key words: sprinkler irrigation, coffee, water balance

\footnotetext{
Projeto financiado pelo PNP\&D/Café e CAPES

2 Doutorando em Engenharia de Água e Solo pela UFLA Campus Universitário, CEP 37200-000, Lavras, MG, Fone: (35) 3829-1362. E-mail: engcarlinhos@hotmail.com; lalima@ufla.br

${ }^{4}$ Escola de Agronomia e Engenharia de Alimentos/UFG. Campus Samambai, CP 131, CEP 74001-970, Goiânia, GO. E-mail: awpego@ufla.br

Engenheira Agrícola, Pesquisadora. Bolsista do CNP\&D/Café, junto ao DEG/UFLA. E-mail: carla@ufla.br
} 


\section{INTRODUÇÃO}

Reconhecidamente, o cafeeiro é afetado pela seca com consequente redução da produção, tornando-se necessária a irrigação, que tem sido utilizada com o propósito de estimular o desenvolvimento vegetativo do cafeeiro, aumentar a produção e obter grãos e bebida de melhor qualidade. $\mathrm{O}$ conhecimento de como as plantas utilizam a água no solo e como respondem aos níveis de disponibilidade a partir do balanço hídrico, pode ser uma saída viável para o estabelecimento de estratégias eficazes de manejo visando ao melhor uso possível das reservas de água no solo pelas culturas.

$\mathrm{Na}$ agricultura irrigada é fundamental o conhecimento das necessidades hídricas das culturas. O consumo de água de uma cultura depende diretamente da demanda energética atmosférica, do conteúdo de água no solo e da resistência da planta à perda de água para a atmosfera. No que se refere ao manejo de irrigação a base para a quantificação da água a ser aplicada em determinada cultura está associada, comumente, à capacidade da superfície do solo e da vegetação de perder água para a atmosfera. A forma usual de se quantificar a água a ser aplicada ao longo do ciclo da cultura é considerar os processos de evaporação do solo e de transpiração da planta, conjuntamente, ao que se denomina evapotranspiração (Silva \& Rao, 2006).

A evapotranspiração pode ser definida através de medidas diretas ou por modelos que levem em consideração a utilização de elementos meteorológicos; no primeiro grupo são utilizados diversos tipos de lisímetro além do método de balanço de água no solo; já no segundo grupo se usam modelos teóricos, empíricos e evaporímetros, como o Tanque Classe "A", ou seja, processos controlados pelo suprimento de água às plantas e pela disponibilidade de energia resultante da interação com as variáveis meteorológicas que condicionam a demanda atmosférica (Pivetta et al., 2010).

O método Penman-Monteith (FAO) é uma referência internacional e representa uma cultura hipotética que permite a obtenção de resultados confiáveis do potencial de evapotranspiração de um local. Segundo Sousa et al. (2010), a ETo é uma variável relevante para o planejamento de irrigação, por ser afetada apenas pelos fatores climáticos. Em geral, os métodos de estimativa da ETo são baseados em variáveis climáticas, muitos deles na temperatura, radiação ou métodos combinados (radiação e temperatura).

Apesar da existência de diversos modelos para se estimar a ETo, eles são utilizados em condições climáticas e agronômicas muito diferentes daquelas em que, inicialmente, foram concebidos e, por isto, é de extrema importância avaliar o grau de exatidão desses modelos antes de utilizá-los para nova condição (Silva et al., 2005; Sousa et al., 2010).

Um procedimento mais eficaz estaria em conduzir o processo de estimativa da evapotranspiração em uma só etapa, descartando-se a utilização dos coeficientes de cultura. Sugerese, então, que o método de Penman-Monteith seja adotado como padrão, com a utilização de valores adequados de resistência aerodinâmica e de resistência do dossel, específicas para cada cultura. Entretanto, a adoção imediata desse método incorreria em algumas dificuldades na obtenção de valores confiáveis para a resistência do dossel (Heldwein et al., 2004; Leitão et al., 2007).

De acordo com Lima (2006), a evapotranspiração média diária para a região de Lavras, nas estações de outono, inverno e primavera de 2003, foi de 2,77, 3,24 e 4,21 mm, respectivamente. O cafeeiro Catuaí (Coffea arabica L.) apresentou, após quatro anos de recepa, entre os meses de abril e setembro de 2004, evapotranspiração de cultura (ETc) variando entre 1,23 mm dia-1 no mês de julho, de 4,82 mm dia-1 no mês de setembro e Kc variando de 0,59 a 1,16 (Sato et al. 2007)

Em geral, nos trabalhos com irrigação em cafeeiro o cálculo da lâmina de irrigação tem sido baseado na evaporação do tanque Classe A, em que os resultados obtidos nas regiões Sul e Triângulo do estado de Minas Gerais indicam que lâminas variando de 60 a $100 \%$ da evaporação do tanque Classe A são as que proporcionam maiores produtividades; sabe-se, porém, que a adoção de um único valor para Kc pode levar, dependendo da época do ano, ao excesso de irrigação ou ao déficit hídrico, ambos prejudiciais às plantas.

$\mathrm{Na}$ tentativa de detalhar o consumo de água do cafeeiro, Oliveira et al. (2003) determinaram valores de Kc que variaram de 0,72 até 1,50 no período de junho a setembro, para lavoura adulta.

Ainda são poucas as informações sobre o melhor suprimento de água no cafeeiro e não existem critérios definitivos de manejo da irrigação, no que se refere a dois fatores: quando irrigar (turno de rega fixo ou variável) e quanto irrigar (lâmina total necessária).

Lima et al. (2008), demonstraram que a irrigação contribui significativamente para o aumento de produtividade, testando diferentes lâminas de irrigação com base nos porcentuais ECA, para cinco safras estudadas e encontraram um acréscimo de $119 \%$ na produtividade do cafeeiro irrigado (lâmina de $60 \%$ da ECA) quando comparada com a do tratamento não irrigado. Rezende et al. (2006) conduziram experimento no município de Lavras com a cultivar Topázio MG-1190, em que foram avaliadas lâminas de irrigação de $0 \%$ (L0 sem irrigação), 40\% (L1), 80\% (L2) e $120 \%$ (L3) da evaporação do Tanque Classe A (ECA) das safras de 2002/2003 e 2003/2004. Os valores da produtividade acumulada apresentaram superioridade para os tratamentos irrigados, com acréscimos em relação ao não irrigado, variando de 23,68\% (L1) a 68,23\% (L2), comparando-se com o não irrigado.

Segundo Silva et al. (2003), os benefícios da irrigação para uma cultura só podem ser alcançados em toda a sua plenitude quando o sistema de irrigação for utilizado com critérios de manejo que resultem em aplicações de água em quantidades compatíveis com as necessidades de consumo da cultura. Ressalta-se que qualquer estratégia de manejo de irrigação deve ser alicerçada nas curvas de consumo de água das culturas e que, para determinação do momento exato para efetuar a irrigação, torna-se necessário racionalizar o manejo de água na agricultura irrigada.

Desta forma se objetivou, neste trabalho, estimar a evapotranspiração e o coeficiente $\mathrm{Kc}$ em diferentes períodos, através do método do balanço hídrico, do cafeeiro "Rubi" irrigado por pivô central. 


\section{Material e MÉTODOS}

O experimento foi desenvolvido na área experimental do Departamento de Engenharia da Universidade Federal de Lavras (DEG-UFLA), em uma lavoura de café irrigada por pivô central. A lavoura estudada se situa na região sul do estado de Minas Gerais, a $21^{\circ} 13^{\prime}$ de latitude sul e $44^{\circ} 58^{\prime}$ de longitude oeste, em uma altitude média de $918,8 \mathrm{~m}$. A temperatura média anual do ar em Lavras, MG, é de $19,4{ }^{\circ} \mathrm{C}$ e o total anual de precipitação pluvial, de $1530 \mathrm{~mm}$ (Brasil, 1992). A variedade do cafeeiro cultivado na área é a "Rubi", plantada deste março de 1999, no espaçamento de 3,5 m entre linhas e $0,80 \mathrm{~m}$ entre plantas. Aárea total do experimento é de aproximadamente 1,6 ha, dividida em 18 talhões com aproximadamente $888 \mathrm{~m}^{2}$ cada uma, visando distribuir os tratamentos. O solo da área é classificado como Latossolo Roxo eutroférrico; a metodologia empregada para determinar o consumo de água do cafeeiro consistiu na determinação do coeficiente da cultura $(\mathrm{Kc})$ para as diferentes fases fenológicas da cultura. Para isto foi realizado um balanço de água no solo, na região do sistema radicular da planta.

O delineamento experimental adotado foi o de blocos casualizados com seis tratamentos e três repetições. Os tratamentos corresponderam às lâminas de água aplicadas em função de percentagens de valores de Kc e ainda utilizados por irrigantes na região e da evapotranspiração de referência (ET0), ou seja: $\operatorname{Tr} 01=0 ; \operatorname{Tr} 02=60 \% \mathrm{Kc}, \operatorname{Tr} 03=80 \% \mathrm{Kc}, \operatorname{Tr} 04=$ $100 \%$ Kc, Tr05 $=120 \%$ Kc e Tr06 $=140 \%$ Kc, totalizando, assim, 18 parcelas. Em cada parcela foram consideradas oito plantas úteis, conforme visualizado na Figura 1, sendo que I, II e III correspondem às repetições 1,2 e 3 , respectivamente.

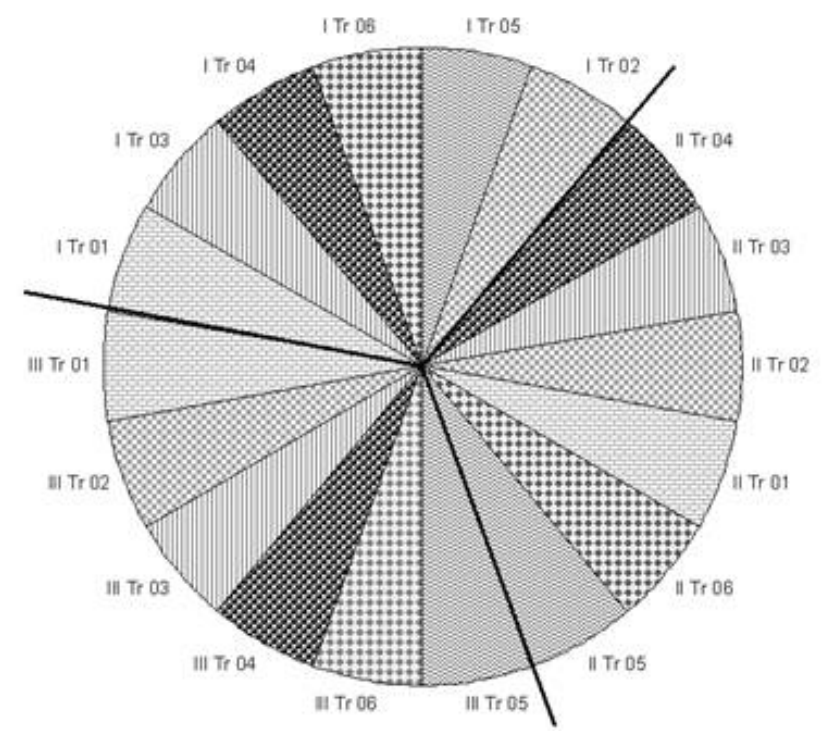

Obs.: $\operatorname{Tr} 01=0$; $\operatorname{Tr} 02=60 \% \mathrm{Kc}$, Tr03 $=80 \%$ Kc, $\operatorname{Tr} 04=100 \%$ Kc, $\operatorname{Tr} 05=120 \% \mathrm{Kc}$ e Tr06 $=140 \% \mathrm{Kc}$

Figura 1. Esquema da área experimental

A coleta e a tabulação dos dados foram realizadas ao longo de dois anos (2007 e 2008). O monitoramento foi dividido em 4 períodos correspondentes às fases fenológicas do café (Tabela 1), a saber:

- Período A - 01/01/08 a 31/03/08 => Kc = 0,90
Tabela 1. Ciclo fenológico do cafeeiro, para as condições do Brasil

\begin{tabular}{llll}
\hline \multicolumn{1}{c}{ Meses } & Estações & Vegetação & \multicolumn{1}{c}{ Fases fenológicas } \\
Jan/Fev/Mar. & Verão & Plena & Granação \\
Abr/Maio/Jun. & Outono & Moderada & Maturação e abotamento \\
Jul/Ago/Set & Inverno & Fraca & Dormência \\
Out./Nov/Dez. & Primavera & Plena & Expansão \\
\hline Fonte: Camargo (1987) & & &
\end{tabular}

- Período B - 01/04/07 a 18/05/07 e 01/04/08 a 18/05/08 => $\mathrm{Kc}=1,30$

$$
\begin{aligned}
& \text { - Período C }-01 / 08 / 07 \text { a 31/10/07 } \Rightarrow \text { Kc }=1,15 \\
& \text { - Período D - 01/11/08 a 31/012/08 } \Rightarrow \text { Kc }=1,10
\end{aligned}
$$

Para comparação dos resultados deve-se observar que o início do experimento não coincidiu com o do ciclo fenológico e, portanto, a produção do ano de 2007 não teve influência total dos tratamentos.

Os principais componentes do balanço de água no solo foram contabilizados em períodos descendias, em um volume de controle de base unitária, que se estende desde a profundidade imediatamente abaixo do sistema radicular da cultura $(0,75 \mathrm{~m})$ até a superfície do solo. Referidos componentes, foram: a precipitação pluvial diária, o escoamento superficial, a irrigação, o armazenamento de água na zona radicular, a drenagem interna, a ascensão capilar e a evapotranspiração da cultura.

Determinou-se a evapotranspiração do cafeeiro (ETc) realizando-se o balanço de água na zona radicular da cultura (Eq. 1), entre os fluxos que contribuem para a adição de água no volume de controle, ou seja, a precipitação pluvial diária, a irrigação e a ascensão capilar, e os componentes que constituem em perdas de água, como o escoamento superficial e a drenagem interna.

$$
\mathrm{ETc}=\mathrm{P}+\mathrm{I}+\mathrm{C}-\mathrm{ES}-\mathrm{D}-\Delta \mathrm{ARM}_{\mathrm{z}}
$$

em que:

$$
\begin{aligned}
& \text { ETc- evapotranspiração da cultura, } \mathrm{mm} \\
& \mathrm{P} \text { - precipitação, } \mathrm{mm} \\
& \mathrm{I} \text { - irrigação, mm } \\
& \mathrm{C} \text { - ascensão capilar, mm } \\
& \text { ES - escoamento superficial, } \mathrm{mm} \\
& \mathrm{D} \text { - drenagem interna, mm } \\
& \Delta \mathrm{ARM}_{\mathrm{z}} \text { - variação no armazenamento da camada, } \mathrm{mm}
\end{aligned}
$$

A contabilização da entrada de água por precipitação foi feita com um pluviômetro instalado na área experimental. As medidas de precipitação acumulada em $24 \mathrm{~h}$ foram realizadas às $8 \mathrm{~h}$ da manhã seguinte, a cada dia chuvoso. Para contabilização do escoamento superficial cada parcela experimental foi delimitada por diques de metal com área de 1 $\mathrm{m}^{2}$. Desta forma foram feitas medições do volume de água escoado por gravidade em cada parcela experimental. A lâmina de escoamento superficial foi determinada através da relação entre o volume escoado e a área de cada parcela experimental.

As irrigações foram realizadas três vezes por semana (segunda, quarta e sexta feira), através de um pivô central 
instalado na área (área total), contendo dois vãos que totalizam 67,5 m de extensão e um lance em balanço de quatro metros, perfazendo um raio molhado de $71,5 \mathrm{~m}$. Calcularam-se as lâminas aplicadas por meio da seguinte equação:

$$
\mathrm{LI}=\frac{\% \mathrm{~K}_{\mathrm{c}} \mathrm{ET}_{0}}{\mathrm{Ei}}
$$

em que:
Li - lâmina de irrigação, $\mathrm{mm}$
Kc - coeficiente de cultura
ETo- evapotranspiração de referência (Penman-Monteith), mm Ei - eficiência de irrigação, 0,9

Obteve-se a lâmina de irrigação através de programação do painel do pivô, variando a velocidade de deslocamento em cada segmento de arco.

Os parâmetros, armazenamento de água no solo por camada e a umidade volumétrica (q) por profundidade, foram quantificados utilizando-se curvas de retenção de água no solo. Obtiveram-se os valores de potencial matricial $\left(\mathrm{h}_{\mathrm{m}}\right)$ por meio de sensores matriciais de uso simplificado conectados a dataloggers, cujos dados foram armazenados de hora em hora. Para a coleta dos dados armazenados nos dataloggers utilizouse um microcomputador. $\mathrm{O}$ armazenamento de água no perfil de solo considerado foi determinado pelo método trapezoidal.

$$
\mathrm{ARM}=\int_{0}^{\mathrm{L}} \theta \mathrm{dz} \cong\left(\bar{\theta}\left(\mathrm{t}_{\mathrm{i}}\right)\right) \mathrm{L}
$$

em que:

ARM - Armazenamento de água no solo, mm

$\theta$ - teor de água, base volume médio das camadas de solo, $\mathrm{m}^{3} \mathrm{~m}^{-3}$

L - profundidade do perfil do solo, $\mathrm{mm}$

Os fluxos de drenagem interna ou ascensão capilar no limite inferior do volume de controle foram estimados pela equação de Darcy-Buckingham, ou seja:

$$
\mathrm{q}=-\mathrm{K}(\theta) \frac{\partial \mathrm{h}_{\mathrm{t}}}{\partial \mathrm{z}}
$$

em que:
$\mathrm{q}$ - fluxo de água no solo, $\mathrm{mm} \mathrm{d}^{-1}$
$\mathrm{K}(\theta)$ - condutividade hidráulica do solo não saturado, $\mathrm{mm} \mathrm{d}^{-1}$
h - potencial matricial, mm.c.a
z - coordenada vertical, positiva para baixo, $\mathrm{mm}$

A condutividade hidráulica do solo não saturado $(K(\theta))$ foi determinada pelo método proposto por Mualem (1976), ou seja:

$$
\mathrm{K}(\theta)=\mathrm{K}_{0} \mathrm{w}^{\mathrm{L}}\left[1-\left(1-\mathrm{w}^{\frac{1}{\mathrm{~m}}}\right)^{\mathrm{m}}\right]^{2}
$$

em que:

w - (saturação relativa) pode ser calculada como:

$$
\mathrm{w}=\frac{\theta-\theta_{\mathrm{r}}}{\theta_{\mathrm{s}}-\theta_{\mathrm{r}}}
$$

$\mathrm{K}_{0}$ é a condutividade hidráulica do solo saturado e L é um parâmetro empírico, estimado por Mualem (1976) como sendo aproximadamente igual a 0,5 para a maioria dos solos (Libardi, 1999). A condutividade hidráulica do solo saturado $\left(K_{0}\right)$ foi determinada no campo utilizando-se o permeâmetro de Guelph.

Estimaram-se, ao final de cada fase fenológica do café, os valores de Kc com base na seguinte equação:

$$
\mathrm{K}_{\mathrm{c}}=\frac{\mathrm{ET}_{\mathrm{c}}}{\mathrm{ET}_{0}}
$$

em que:

Kc - coeficiente de cultura, mm

ETc - evapotranspiração da cultura, $\mathrm{mm}$

$\mathrm{ET}_{0}$ - evapotranspiração de referência, mm

A evapotranspiração de referência $\left(\mathrm{ET}_{0}\right)$ foi estimada por dois métodos distintos, o do tanque Classe A e o de PenmanMonteith padrão FAO 1998, conforme Allen et al. (1998), Eqs. 7 e 7.1,

$$
\mathrm{ET}_{0}=\mathrm{k}_{\mathrm{p}} \mathrm{EV}
$$

donde:

$$
\mathrm{k}_{\mathrm{p}}=0,482+0,024 \ln (\mathrm{F})-0,000376 \mathrm{U}_{2}+0,0045 \mathrm{UR}
$$

em que:

$\mathrm{ET}_{0}$ - evapotranspiração de referência, $\mathrm{mm} \mathrm{d}^{-1}$

$\mathrm{Kp}$ - coeficiente do tanque A

$\mathrm{EV}$ - evaporação do tanque, $\mathrm{mm} \mathrm{d}^{-1}$

$\mathrm{F}$ - distância da área de bordadura, $\mathrm{m}$

$\mathrm{U}_{2}$ - velocidade média do vento a $2 \mathrm{~m}$ de altura, $\mathrm{km} \mathrm{d}^{-1}$

UR - umidade relativa, \%

$$
\mathrm{ET}_{0}=\frac{\mathrm{s}}{\left(\mathrm{s}+\gamma^{*}\right)}\left(\mathrm{R}_{\mathrm{n}}-\mathrm{G}\right) \frac{1}{\lambda}+\frac{\gamma}{\left(\mathrm{s}+\gamma^{*}\right)} \frac{900}{(\mathrm{~T}+273)} \mathrm{U}_{2}\left(\mathrm{e}_{\mathrm{s}}-\mathrm{e}_{\mathrm{a}}\right)(8)
$$

em que:

s - declividade da curva de pressão de saturação de vapor, $\mathrm{kPa}^{\circ} \mathrm{C}^{-1}$

$\mathrm{R}_{\mathrm{n}}$ - saldo de radiação, $\mathrm{MJ} \mathrm{m}^{-2} \mathrm{~d}^{-1}$

$\mathrm{G}$ - fluxo de calor no solo, $\mathrm{MJ} \mathrm{m}^{-2} \mathrm{~d}^{-1}$

$\lambda$ - calor latente de evaporação, $\mathrm{MJ} \mathrm{kg}^{-1}$

$\mathrm{e}_{\mathrm{a}}$ - pressão parcial de vapor, $\mathrm{kPa}$

$\mathrm{e}_{\mathrm{s}}$ - pressão de saturação de vapor, $\mathrm{kPa}$

$\gamma$ - coeficiente psicrométrico, $\mathrm{kPa}^{\circ} \mathrm{C}^{-1}$

$\gamma^{*}$ - coeficiente psicrométrico modificado, $\mathrm{kPa}^{\circ} \mathrm{C}^{-1}$ 
$\mathrm{T}$ - temperatura média do $a r,{ }^{\circ} \mathrm{C}$

$\mathrm{U}_{2}$ - velocidade média do vento a $2 \mathrm{~m}$ de altura, $\mathrm{m} \mathrm{s}^{-1}$

Os elementos meteorológicos necessários à estimativa da $\mathrm{ET}_{0}$ durante a realização do experimento foram obtidos na estação principal do INMET (Instituto Nacional de Meteorologia), localizada a $300 \mathrm{~m}$ da área experimental.

Ressalta-se, para a realização do balanço de água no solo, que se utilizaram os dados de $\mathrm{ET}_{0}$ estimados pelo método de Penman-Monteith (PM) considerado, por pesquisadores da FAO, método padrão (Allen et al., 1998). Os dados estimados pelo método do tanque Classe A foram comparados com os obtidos pelo método de Penman-Monteith (PM), visando à sua utilização por produtores irrigantes da região, pela sua simplicidade, tal como para determinação do produto entre Kp e Kc.

\section{RESULTADOS E DISCUSSÃO}

\section{Evapotranspiraçãode referência $\left(\mathbf{E T}_{0}\right)$}

Os valores médios de evapotranspiração de referência $\left(\mathrm{ET}_{0}\right)$, estimados pelos métodos do Tanque Classe A (TCA) e de Penmam-Monteith (PM), para os períodos 01/04 a 31/07 (B), 01/08 a 31/10 (C) e 01/11 a 31/12 (D) do ano de 2007 e para os períodos de 01/01 a 31/03 (A) e de 01/04 a 18/05 (B) do ano de 2008, com o propósito de se ter um dos parâmetros necessários a determinação do coeficiente de cultura $(\mathrm{Kc})$, podem ser observados na Tabela 2 .

Tabela 2. Valores médios da evapotranspiração diária de referência $\left(\mathrm{ET}_{0}\right)$, estimada pelos métodos do tanque Classe A e Penmam-Monteith para a cidade de Lavras, MG, 2007 e 2008

\begin{tabular}{|c|c|c|c|c|c|c|}
\hline \multirow{2}{*}{ Períodos } & \multirow{2}{*}{ Meses } & \multirow{2}{*}{$\begin{array}{l}\text { Tmed } \\
\left({ }^{\circ} \mathrm{C}\right)\end{array}$} & \multirow{2}{*}{$\begin{array}{l}\text { UR } \\
\text { (\%) }\end{array}$} & \multirow{2}{*}{$\begin{array}{c}\mathbf{V} \\
\left(\mathrm{m} \mathrm{s}^{-1}\right)\end{array}$} & \multicolumn{2}{|c|}{$\begin{array}{l}\text { Média de ETo } \\
\text { diária }\left(\mathrm{mm} \mathrm{d}^{-1}\right)\end{array}$} \\
\hline & & & & & $\begin{array}{l}\text { ETO } \\
\text { (TCA) }\end{array}$ & $\begin{array}{l}\mathrm{ET}_{0} \\
\text { (PM) }\end{array}$ \\
\hline \multirow{4}{*}{ B } & abr/07 & 21,86 & 72,40 & 2,17 & \multirow{4}{*}{3,3} & \multirow{4}{*}{3,2} \\
\hline & mai/07 & 18,09 & 70,77 & 2,21 & & \\
\hline & jun/07 & 17,26 & 66,29 & 2,18 & & \\
\hline & $\mathrm{jul} / 07$ & 17,06 & 66,85 & 2,21 & & \\
\hline \multirow{3}{*}{ C } & ago/07 & 18,93 & 55,23 & 2,66 & \multirow{3}{*}{4,5} & \multirow{3}{*}{4,4} \\
\hline & set/07 & 21,60 & 52,37 & 3,12 & & \\
\hline & out/07 & 22,74 & 59,21 & 2,70 & & \\
\hline \multirow{2}{*}{ D } & nov/07 & 21,51 & 77,52 & 2,81 & \multirow{2}{*}{4,2} & \multirow{2}{*}{4,2} \\
\hline & $\mathrm{dez} / 07$ & 22,99 & 72,93 & 2,49 & & \\
\hline \multirow{3}{*}{ A } & $\mathrm{jan} / 08$ & 21,84 & 78,16 & 2,57 & \multirow{3}{*}{3,8} & \multirow{3}{*}{3,7} \\
\hline & fev/08 & 22,09 & 79,76 & 2,06 & & \\
\hline & $\mathrm{mar} / 08$ & 21,86 & 76,97 & 2,27 & & \\
\hline \multirow{2}{*}{ B } & $\mathrm{abr} / 08$ & 20,82 & 80,00 & 2,00 & \multirow{2}{*}{2,8} & \multirow{2}{*}{2,7} \\
\hline & mai/08 & 17,04 & 75,15 & 2,34 & & \\
\hline
\end{tabular}

Observando a Tabela 2 verificou-se, através dos dados de umidade relativa média do ar no período de coleta e análise dos dados referentes aos meses de abril de 2007 a maio de 2008, que o valor médio decresceu de 72,4 a $52,37 \%$, no outono/ inverno de 2007.
A temperatura média variou de 17,04 a $22,99^{\circ} \mathrm{C}$, com baixa contribuição para o processo de evapotranspiração porém como a média anual da temperatura durante a realização do experimento de março 2007 a maio de 2008 ficou em $20,4{ }^{\circ} \mathrm{C}$, conforme o zoneamento climático realizado pelo Consórcio Brasileiro de Pesquisa e Desenvolvimento do Café, CBPDC (2007), verificam-se condições favoráveis de temperatura para o desenvolvimento fisiológico do cafeeiro suprindo-se, desta forma, as demandas hídricas e nutricionais.

Comparando-se os valores de $\mathrm{ET}_{0}$ estimados por meio das duas metodologias propostas (TCA e PM), conclui-se que os valores estimados pelo método do tanque Classe A se aproximaram dos obtidos pelo método de Penmam-Monteith em todos os períodos estudados, com pequena tendência de superestimativa dos valores de $\mathrm{ET}_{0}$ estimados pelo método do tanque Classe A

$\mathrm{Na}$ Tabela 2 estão reunidos os dados médios de evapotranspiração de referência $\left(\mathrm{ET}_{0}\right)$. Com relação à $\left(\mathrm{ET}_{0}\right)$, observa-se que nos meses de agosto, setembro e outubro de 2007, que correspondem ao período $\mathrm{C}$, os valores médios foram mais elevados quando comparados com o restante do ano, alcançando 4,5 para (TCA) e $4,4 \mathrm{~mm} \mathrm{~d}^{-1}$ para (PM), o que demanda maior quantidade de água pelas plantas. Por outro lado, o período B se sobressaiu em relação aos demais, alcançando os menores valores de $\mathrm{ET}_{0}$ dentre os períodos avaliados. De acordo com os dados apresentados por Lima (2006), a média alcançada nesses meses está acima daquela encontrada pelo autor, o qual cita um valor de $3,86 \mathrm{~mm} \mathrm{~d}^{-1}$ por (PM).

\section{Evapotranspiração da cultura (ETc)}

Os valores médios de evapotranspiração da cultura do cafeeiro (ETc) verificados para os períodos 01/04 a 31/07 (B), 01/08 a 31/10 (C) e 01/11 a 31/12 (D) do ano de 2007 e para os períodos de 01/01 a 31/03 (A) e de 01/04 a 18/05 (B) do ano de 2008, são visualizados na Tabela 3 .

Tabela 3. Valores médios da evapotranspiração da cultura do cafeeiro nos diferentes períodos de irrigação

\begin{tabular}{cccccc}
\hline \multirow{2}{*}{ Períodos } & \multicolumn{5}{c}{ ETc para os períodos de irrigação $\left(\mathbf{m m ~ d}^{-\mathbf{1}}\right)$} \\
\cline { 2 - 6 } & Tr 02 & Tr 03 & Tr 04 & Tr 05 & Tr 06 \\
B & 2,53 & 2,94 & 3,46 & 4,19 & 4,57 \\
C & 5,04 & 5,96 & 7,14 & 8,20 & 9,25 \\
D & 4,85 & 5,46 & 5,41 & 5,80 & 5,87 \\
A & 4,42 & 5,19 & 5,57 & 6,13 & 6,47 \\
B & 2,13 & 2,51 & 3,07 & 3,34 & 4,05 \\
\hline Média & 3,80 & 4,41 & 4,93 & 5,53 & 6,04 \\
\hline
\end{tabular}

A maior taxa de evapotranspiração diária média foi obtida no período 01/08 a 31/10 (C) que corresponde ao estádio florada e expansão dos frutos com 5,4; 5,96; 7,14; 8,20 e 9,25 $\mathrm{mm} \mathrm{d}^{-1}$, respectivamente, para os tratamentos $\operatorname{Tr} 02, \operatorname{Tr} 03, \operatorname{Tr}$ $04, \operatorname{Tr} 05$ e $\operatorname{Tr} 06$. Obtiveram-se as menores taxas de evapotranspiração diária média no período B (01/04 a 31/07 e 01/04 a 18/05), estádio de maturação dos frutos, com valores de 2,$3 ; 2,51 ; 3,07 ; 3,34$ e 4,05 $\mathrm{mm} \mathrm{d}^{-1}$, nos tratamentos Tr 02 , Tr 03, Tr 04, Tr 05 e Tr 06, respectivamente; a evapotranspiração média diária do ciclo produtivo do cafeeiro nos tratamentos 
$\operatorname{Tr} 02, \operatorname{Tr} 03, \operatorname{Tr} 04, \operatorname{Tr} 05$ e $\operatorname{Tr} 06$ foi de 3,80; 4,41; 4,93; 5,53 e $6,04 \mathrm{~mm}$, respectivamente.

Portanto, o tratamento T6 foi o que apresentou a maior taxa de evapotranspiração média diária, tanto no período total quanto nos estádios fenológicos; assim, a ETc aumentou em função do aumento da lâmina de irrigação e os resultados apresentados indicam que a evapotranspiração do cafeeiro foi bastante influenciada pelo volume de água aplicado ao solo como era previsto.

Com base na Tabela 3 e se considerando o tratamento Tr 04 $(100 \% \mathrm{Kc})$ aquele que os agricultores da região utilizam para o cálculo da lâmina de irrigação, os valores da evapotranspiração da cultura (ETc) do café determinada pelo balanço de água no solo foram de 3,46, 7,14 e 5,41, para os períodos 01/04 a 31/07 (B), 01/08 a 31/10 (C) e 01/11 a 31/12 (D) do ano de 2007, respectivamente, enquanto para os períodos de 01/01 a 31/03 (A) e de 01/04 a 18/05 (B) do ano de 2008 os valores de ETc foram de 5,57 e 3,07, respectivamente, e um valor médio de 4,93 mmd-1.

Mantovani (2001) obteve, como resultados de ETc do cafeeiro irrigado por pivô central nas regiões Norte do Espírito Santo e Extremo Sul da Bahia, em fase de produção, valores da ordem de 0,6 a $1,0 \mathrm{~mm}$ d- 1 no período de menor demanda atmosférica e de 4,5 a 4,8 mm d-1 no período de maior demanda. Os valores de consumo de água observados por esses autores foram inferiores aos obtidos neste experimento.

\section{Coeficiente de cultura $(\mathrm{Kc})$}

Os valores médios dos coeficientes de cultura $(\mathrm{Kc})$ para os diferentes períodos analisados utilizando-se o método de Penman-Monteith no cálculo da evapotranspiração de referência $\left(\mathrm{ET}_{0}\right)$, podem ser observados na Tabela 4 .

Tabela 4. Valores médios dos coeficientes de cultura (Kc) do cafeeiro nos diferentes estádios fenológicos, para os tratamentos que receberam irrigação

\begin{tabular}{cccccc}
\hline \multirow{2}{*}{ Períodos } & \multicolumn{5}{c}{ Kc para os períodos de irrigação } \\
\cline { 2 - 6 } & Tr 02 & Tr 03 & Tr 04 & Tr 05 & Tr 06 \\
B & 0,79 & 0,92 & 1,08 & 1,31 & 1,42 \\
C & 1,14 & 1,34 & 1,61 & 1,84 & 2,06 \\
D & 1,08 & 1,23 & 1,25 & 1,44 & 1,46 \\
A & 1,16 & 1,24 & 1,32 & 1,40 & 1,44 \\
B & 0,71 & 0,86 & 1,04 & 1,17 & 1,37 \\
\hline Média & 0,98 & 1,12 & 1,26 & 1,43 & 1,55 \\
\hline
\end{tabular}

Verificou-se decréscimo nos valores de Kc do período A (01/01 a 31/03) para o período B (01/04 a 18/05), os quais coincidem com a fase fenológica de maturação/abotoamento, caracterizada por moderada atividade vegetativa. A partir de agosto ocorreu aumento nos valores de Kc, que corresponde ao período C (01/08 a 31/10), fato associado à atividade vegetativa plena e à fase fenológica de expansão.

Os valores de Kc encontrados considerando-se o tratamento $\operatorname{Tr} 04(100 \% \mathrm{Kc})$, como o tratamento que agricultores da região utilizam para o cálculo da lâmina de irrigação para os períodos 01/04 a 31/07 (B), 01/08 a 31/10 (C) e 01/11 a 31/12 (D) do ano de 2007 foram de 1,08, 1,61 e 1,25, respectivamente. Entretanto, para os períodos de 01/01 a 31/03 (A) e de 01/04 a 18/05 (B) do ano de 2008 os valores de Kc do cafeeiro para os períodos A e B foram de 1,32 e 1,04. Observou-se, para o período B, que os valores de $\mathrm{Kc}$ analisados nos dois anos foram praticamente iguais $(1,08$ e 1,04; média = 1,06), (Tabela 3$)$.

Assim, nos períodos analisados os valores de Kc encontrados foram maiores do que os utilizados para o cálculo da lâmina de irrigação por agricultores da região $(1,10 ; 0,90$; 1,30; e 1,15). Entretanto, tais valores se aproximam dos recomendados por Rocha et al. (2006) que encontraram, para a cultivar Catuaí, cultivada em Planaltina, DF, valores de Kc nos períodos de junho a agosto e de setembro a maio, para cafeeiros com mais de dois anos de idade, valores de 1,0 e 1,25, respectivamente.

Os valores encontrados neste trabalho diferem um pouco dos determinados por Oliveira et al. (2003), que encontraram valores de Kc variando entre 0,72 a 1,50 , no período de junho a setembro, para lavoura adulta. Por outro lado, os valores de Kc encontrados na literatura são considerados constantes, dependendo apenas da idade, isto é, sem se levar em consideração a fenologia da cultura, haja vista que, normalmente, esses valores máximos são utilizados nos dimensionamentos de sistemas de irrigação; por outro lado, através dos resultados obtidos neste estudo se verificam variações nos valores de Kc em resposta às condições do clima local, ao manejo da irrigação e à demanda de água pelo cafeeiro, evidenciando a importância da obtenção do Kc nas diferentes fases fenológicas do cafeeiro.

\section{Conclusão}

Os valores de Kc do cafeeiro encontrados para o cálculo da lâmina de irrigação para os períodos períodos A - 01/01/08 a 31/ 03/08; B - 01/04/07 a 18/05/07 e 01/04/08 a 18/05/08; C - 01/08/07 a 31/10/07 e D - 01/11/08 a 31/012/08, foram de 1,32, 1,06, 1,61, 1,25 , respectivamente.

\section{Agradecimentos}

Ao Consórcio Nacional de Pesquisa do Café, que fomentou este trabalho.

\section{LITERATURA CITADA}

Allen, T. G.; Pereira, L. S.; Raes, D.; Smith, M. Crop evapotranspiration: guidelines for computing crop water requirements. Roma: FAO, 1998. 300p. Irrigation and Drainage, Paper 56

Brasil - Ministério da Agricultura e Reforma Agrária. Secretaria Nacional de Irrigação.Normais Climatológicas: 1961-1990. Brasília, 1992. 84p.

CBPDC - Consórcio Brasileiro de Pesquisa e Desenvolvimento do Café. Zoneamento climático da cultura do café (Coffea arabica L.) no estado de Minas Gerais. <http:// www.cpa.unicamp.br/ cafe/MG_menu.html>. 15 Jun. 2007. 
Heldwein, A. B.; Dalmago, G. A.; Streck, L.; Tazzo, I. F.; Trentin, G. Utilização do evaporímetro de Piche exposto à radiação solar para estimar a evapotranspiração máxima do pimentão em estufa plástica. Revista Brasileira de Agrometeorologia, v.9, p.213-217, 2004.

Leitão, M. de M. V. B. R.; Oliveira, G. M. de; Leitão, T. J. V. Avaliação do desempenho de diferentes métodos de estimativa da evaporação para duas regiões da Paraíba. Revista Brasileira de Engenharia Agrícola e Ambiental, v.11, p.585-593, 2007.

Libardi, P. L. Dinâmica da água no solo. 2.ed. Piracicaba: ESALQ/USP, 1999. 497p.

Lima, E. P. Graus-dia, temperatura base e coeficientes de cultura para cafeeiros arábicaem fase de implantação. Lavras: UFLA, 2006. 116p. Tese Doutorado

Lima, L. A.; Custódio, A. A. P de; Gomes, N. M. Produtividade e rendimento do cafeeiro nas cinco primeiras safras irrigado por pivô central em Lavras, MG. Ciência e Agrotecnologia, v.32,p.1832-1842, 2008

Mantovani, E. C. A Irrigação do cafeeiro. Item, Irrigação e Tecnologia, n.48, p.45-49, 2001.

Mualem, Y. A new model for predicting the hydraulic conductivity of unsaturated porous media. Water Resource Research, v.12, p.513-522, 1976.

Oliveira, P. M. de ; Silva, A. M. da; Castro Neto, P. Estimativa da evapotranspiração e do coeficiente de cultura do cafeeiro (coffea arabica 1.). Irriga, v.8, p.273-282, 2003.

Pivetta, C. R.; Heldwein, A. B.; Maldaner, I. C.; Radons, S. R.; Tazzo, I. F.; Lucas, D. D. Evapotranspiração máxima do pimentão cultivado em estufa plástica em função de variáveis fenométricas e meteorológicas. Revista Brasileira de Engenharia Agrícola e Ambiental, v. 14, p.768-775, 2010.
Rezende, F. C,; Oliveira, S. R.; Faria, M. A.; Resende, K. A. Características produtivas do cafeeiro (Coffea arabica L. cv., topázio mg-1190), recepado e irrigado por gotejamento. Coffee Science, v.1, p.103-110, 2006.

Rocha, O. C.; Guerra, A.F.; Silva, F.A.M.; Machado Júnior, J. R. R.; Araujo, M. C.; Silva, H. H. Programa para monitoramento de irrigação do cafeeiro no cerrado. In: Simpósio Brasileiro de Pesquisa em Cafeicultura Irrigada, 8, 2006, Araguari. Anais... Araguari: ACA, 2006. p.61-64.

Santinato, R.; Fernandes, A. L. T.; Fernandes, D. R. Irrigação na cultura do café. Campinas: Arbore, 1996. 146p.

Sato, F. A.; Silva, A. M. da; Coelho, G.; Silva, A. C. da; Carvalho, L. G. de. Coeficiente de cultura (Kc) do cafeeiro (Coffea arabica L.) no período de outono-inverno. Engenharia Agrícola, v.27, p.383-391, 2007.

Silva, L. C.; Rao, T. V. R. Avaliação de métodos para estimativa de coeficientes da /cultura de amendoim. Revista Brasileira de Engenharia Agrícola e Ambiental, v.10, p.128-131, 2006.

Silva, M. L. O. E.; Faria, M. A.; Garcia, J. R. S.; Borges, C. T. Produtividade das quatro primeiras safras do cafeeiro (Coffea arabica L.) sob diferentes lâminas de irrigação e fertirrigação. In: Congresso Brasileiro de Engenharia Agrícola, 32, 2003, Goiânia. Anais... Jaboticabal: SBEA, 2003, CD-Rom.

Silva, V. de P. R. da; Belo Filho, A. F.; Silva, B. B. da; Campos, J. H. B. da C. Desenvolvimento de um sistema de estimativa da evapotranspiração de referência. Revista Brasileira de Engenharia Agrícola e Ambiental, v.9, p.547-553, 2005.

Sousa, I. F. de; Silva, V. P. R. da; Sabino, F. G.; Netto, A. de O.; Silva, B. K. N.; Azevedo, P. V. Evapotranspiração de referência nos perímetros irrigados do estado de Sergipe. Revista Brasileira de Engenharia Agrícola e Ambiental, v.14, p.633-644, 2010. 